\title{
TITLE:
}

\section{Self-sustained spin precession in an optical tristable system}

AUTHOR(S):

Kitano, M.; Yabuzaki, T.; Ogawa, T.

\section{CITATION:}

Kitano, M. ... [et al]. Self-sustained spin precession in an optical tristable system. Physical Review A 1981, 24(6): 3156-3159

\section{ISSUE DATE:}

1981-12

URL:

http://hdl.handle.net/2433/152446

RIGHT:

(C) 1981 The American Physical Society 


\title{
Self-sustained spin precession in an optical tristable system
}

\author{
M. Kitano, T. Yabuzaki, and T. Ogawa \\ Radio Atmospheric Science Center, Kyoto University, Uji 611, Japan
}

(Received 7 July 1981)

\begin{abstract}
The dynamical behavior of a Fabry-Perot cavity filled with atoms having Zeeman sublevels in the ground state is investigated. Such a system is known to exhibit optical tristability, where the atomic spins are oriented parallel or antiparallel to the optical axis even for the linearly polarized incident light. It is found that, when a static magnetic field is applied transversely to the optical axis, self-sustained precession of the spin polarization occurs. Correspondingly, the $\sigma_{+}$and $\sigma_{-}$components of the transmitted light are modulated at about the Larmor frequency. We also show that the Bloch equation describing the motion of the spin polarization in the cavity can be reduced to van der Pol's equation.
\end{abstract}

We have shown in the preceding paper ${ }^{1}$ that a Fabry-Perot cavity containing atoms which have degenerate Zeeman sublevels in the ground state exhibits optical tristablility. The phenomena are due to spin polarization in the ground state by optical pumping in the region of anomalous dispersion. In the case of linearly polarized incident light the three states are: (i) the transmitted light $\left(I_{T}\right)$ and the reflected light $\left(I_{R}\right)$ are both linearly polarized (linear state); (ii) $I_{T}$ is right circularly polarized and $I_{R}$ is left circulary polarized ( $\sigma_{+}$state); and (iii) a state with opposite polarizations to the $\sigma_{+}$state $\left(\sigma_{-}\right.$state). When the incident light intensity exceeds a threshold level, the linear state becomes unstable and symmetry breaking transition to the $\sigma_{+}$or $\sigma_{-}$states occurs. Above the threshold, atomic spins are forced to orient parallel or antiparallel to the optical axis. This phenomenon can be considered as an example of self-circular birefringence proposed by Tam and Happer. ${ }^{2}$

When a static magnetic field transverse to the optical axis is applied, the "spontaneous" magnetization begins to precess about it. In general, precession of macroscopic magnetization is faded out by thermal relaxations unless it is driven by periodic external forces such as a radio-frequency magnetic field or a modulated light beam. ${ }^{3}$ In the present paper we show that self-sustained precession is possible in our system without any periodic external forces. According to the precession the circulary polarized components of the transmitted light are modulated regeneratively. It is a new type of self-pulsing in optical bistability (or multistabilty) and we may call it "magnetically-inducedself-pulsing."
Self-pulsing phenomena in an optical bistable system was proposed and demonstrated experimentally by $\mathrm{McCall}^{4}$ It is explained as a relaxation oscillation due to a medium having two opposing contributions to the nonlinear refractive index, which have different time responses to each other. A similar relaxation oscillation was proposed by Szöke et al., ${ }^{5}$ although its period is determined mainly by cavity holding time. Light propagation effects in a ring cavity with nonlinear medium also induce instabilities, where the pulsing frequency is related to the frequency difference of the cavity modes. ${ }^{6}$ In our system the frequency is determined by the Larmor frequency, namely, by the strength of the static magnetic field.

We consider atoms with energy levels shown in Fig. 1. The atoms are optically pumped by right circularly polarized light $\left(\sigma_{+}\right)$and left circulary polarized light $\left(\sigma_{-}\right)$simultaneously. We can neglect the population of the excited state assuming that the spontaneous decay rate $\gamma_{e}$ is large enough compared to the pumping rates. The time evolution of the ground state $\left(J=\frac{1}{2}\right)$ can be described by the Bloch equation, ${ }^{7}$

$$
\begin{aligned}
\frac{d \overrightarrow{\mathbf{M}}}{d t} & =\gamma \overrightarrow{\mathbf{M}} \times \overrightarrow{\mathrm{H}}_{0}-\Gamma \overrightarrow{\mathrm{M}} \\
& \quad P_{+}\left(\overrightarrow{\mathrm{M}}-\overrightarrow{\mathrm{M}}_{0}\right)-P_{-}\left(\overrightarrow{\mathrm{M}}+\overrightarrow{\mathrm{M}}_{0}\right),
\end{aligned}
$$

where $\gamma$ and $\Gamma$ are the gyromagnetic ratio and the spin relaxation rate of the ground state, respectively. The third (fourth) term of the right-hand side of Eq. (1) represents the effect of optical pumping by the $\sigma_{+}\left(\sigma_{-}\right)$component of the intracavity light.

3156 (c)1981 The American Physical Society 


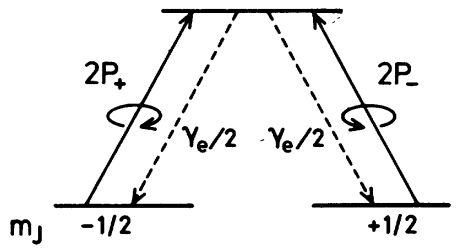

FIG. 1. Simplified atomic level scheme. Optically excited states are represented by a single level bý assuming that the collisional mixing among these states is complete.

If the pumping rate $\boldsymbol{P}_{+}\left(\boldsymbol{P}_{-}\right)$is large enough, all atoms are pumped to the state $m_{J}=\frac{1}{2}\left(-\frac{1}{2}\right)$ and maximum polarization $\overrightarrow{\mathbf{M}}_{0}\left(-\overrightarrow{\mathrm{M}}_{0}\right)$ along the optical axis is established. The pumping rates $\boldsymbol{P}_{ \pm}$ are expressed in terms of the $\sigma_{+}$and $\sigma_{-}$light intensities (photon flux) $I_{ \pm}$and the absorption cross section $\sigma$ by

$$
P_{ \pm}=\frac{1}{2} \sigma I_{ \pm} \text {. }
$$

For a homogeneously broadened medium, the absorption cross section $\sigma$ for monochromatic light of the frequency $\omega$ is given by

$$
\sigma=\frac{4 \pi d^{2} \omega_{0}}{c \hbar} \frac{\gamma_{a b}}{\Delta^{2}+\gamma_{a b}^{2}},
$$

where $d$ is the atomic dipole moment, $\gamma_{a b}$ is the relaxation rate for the optical coherence, $\omega_{0}$ is the optical transition frequency, and $\Delta=\omega-\omega_{0}$ is the atomic detuning. For simplicity we neglected effects of the static magnetic field $H_{0}$ on $\sigma$ assuming the Zeeman frequency $\Omega_{0}=\gamma H_{0}$ is small compared with the homogeneous width $\gamma_{a b}$.

Taking the $z$ axis along the light propagation direction and the $y$ axis to the direction of the static magnetic field $H_{0}$ (Fig. 2), we obtain the equations for the normalized magnetization components $m_{i}=M_{i} / M_{0}(i=x, y, z):$

$$
\begin{aligned}
\frac{d m_{x}}{d t}= & -\Omega_{0} m_{z}-\left(\Gamma+P_{+}+P_{-}\right) m_{x}, \\
\frac{d m_{y}}{d t}= & -\left(\Gamma+P_{+}+P_{-}\right) m_{y}, \\
\frac{d m_{z}}{d t}= & \Omega_{0} m_{x}-\left(\Gamma+P_{+}+P_{-}\right) m_{z} \\
& +\left(P_{+}-P_{-}\right) .
\end{aligned}
$$

From Eq. (4b) we see that $m_{y}$ decays sooner or later, so hereafter we concern ourselves only with Eqs. (4a) and (4c).

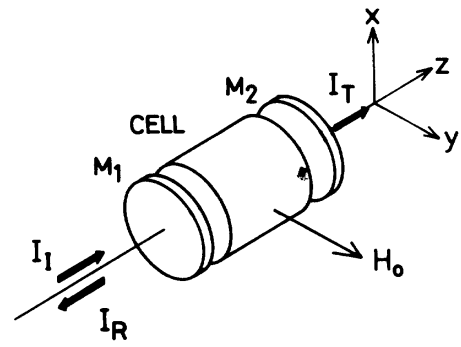

FIG. 2. Fabry-Perot cavity with nonlinear medium cell. Static magnetic field $H_{0}$ is applied along the $y$ axis.

The susceptibilities for $\sigma_{+}$and $\sigma_{-}$light are determined by the magnetization component along their propagation direction, namely, $m_{z} \cdot{ }^{7}$ We assume that $m_{z}$ varies slowly with respect to the cavity damping time $L / c T$, where $L$ is the length of the cavity and $T$ is the transmissivity of the mirrors. Then we can adopt the quasistatic susceptibility approximation. ${ }^{8}$ The absorption coefficients $\alpha_{ \pm}$and the wave numbers $k_{ \pm}$are

$$
\begin{aligned}
& \alpha_{ \pm}\left(m_{z}\right)=\alpha_{0}\left(1 \mp m_{z}\right), \\
& k_{ \pm}\left(m_{z}\right)=k_{0}+\kappa\left(1 \mp m_{z}\right),
\end{aligned}
$$

where $\alpha_{0}$ and $k_{0}+\kappa$ are the absorption coefficient and the wave number for the unpolarized medium. To simplify the situation we neglect the absorption effect by taking relatively large values of $|\Delta|$.

Thus, the transmission characteristics of the Fabry-Prot cavity are given by the equation

$I_{T \pm}=T^{2} I_{I \pm}\left[1+R^{2}-2 R \cos 2 k_{ \pm}\left(m_{z}\right) L\right]^{-1}$,

where $I_{I \pm}$ and $I_{T \pm}$ are the incident and transmitted light intensities, respectively, and $R=1-T$ is the reflectivity of the mirrors. We also assumed that $m_{z}$ has no $z$ dependence due to the standing-wave structure of the pumping field, because the atoms move many wavelengths during the pumping time $P_{ \pm}^{-1}$ or the decay time $\Gamma^{-1}$. The effective pumping light intensity is represented as a sum of the forward and backward wave intensities in the cavity:

$$
I_{ \pm}=I_{T \pm}(1+R) / T \text {. }
$$

By using Eqs. (2), (6), and (7), the pumping rates in Eqs. (4) are represented as follows:

$P_{ \pm}=T\left[1+R^{2}-2 R \cos 2 k_{ \pm}\left(m_{z}\right) L\right]^{-1} Q_{ \pm}$,

where $Q_{ \pm}=\frac{1}{2} \sigma(1+R) I_{I \pm}$ are quantities related to to the incident light intensities. Substituting Eq.

(8) into Eqs. (4a) and (4c), we obtain two- 
dimensional nonlinear differential equations which describe the motion of the Bloch vector $\overrightarrow{\mathbf{m}}=\left(m_{z}, m_{x}\right)$ in the cavity.

Let us consider, at first, the case where no external magnetic field is applied, namely, the case of $\Omega_{0}=0$. Here it is sufficient only to handle Eq. (4c), since Eq. (4a) gives a stable stationary solution $m_{x}=0$. We rewrite Eq. (4c) with $\Omega_{0}=0$ as

$$
\frac{d m_{z}}{d t}=f_{\mu}\left(m_{z}\right)
$$

where $\mu$ represents parameters such as $\Gamma$ and $Q_{ \pm}$ as a whole. The equilibrium points are found by solving the equation $f_{\mu}\left(m_{z}\right)=0$, which is equivalent to Eq. (9) in Ref. 1, although the latter is for the field variables and the former is for the atomic variable. We also find equivalence of $f_{\mu}\left(m_{z}\right)$ to $\partial V / \partial x$ in Ref. 1 , where $V(x)$ is a system potential introduced in context of the catastrophe theory. The stability of an equilibrium point is determined by the first derivative of $f_{\mu}$ with respect to $m_{z}$ at the point. If $f_{\mu}^{\prime}>0$, the equilibrium point is unstable and if $f_{\mu}^{\prime}<0$, then stable. Figure 3 shows some examples of $f_{\mu}\left(m_{z}\right)$ in the case of linearly polarized incident light. We choose the parameters $\mu$ so as to give single-stable, tristable, and bistable cases. We show in Fig. 4 the trajectories of the Bloch vector $\overrightarrow{\mathrm{m}}$ on the $\left(m_{z}, m_{x}\right)$ plane for the bistable case. There the equilibrium

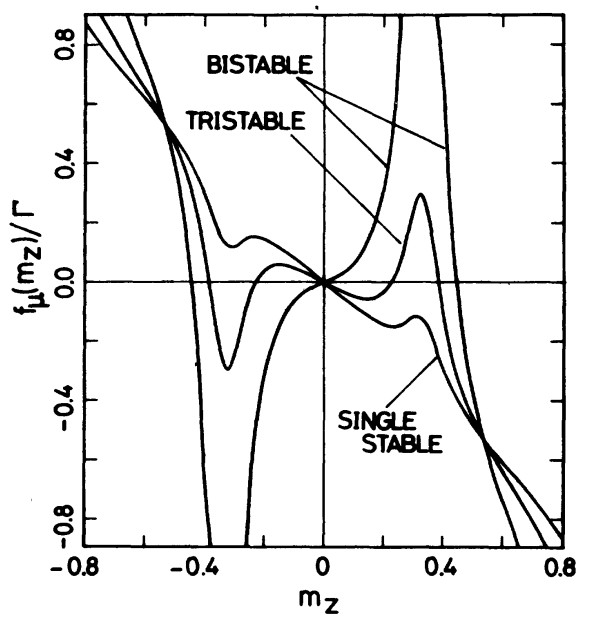

FIG. 3. Plot of the function $f_{\mu}\left(m_{z}\right)$ for single-stable, tristable, and bistable cases. Parameters are $R=0.7$; $2 k_{0} L=-\pi+2 M \pi$ (M: integer); $2 \kappa L=1.5 \pi ;$ and $Q_{+}=Q_{-}=0.1 \Gamma$ (single stable), $Q_{+}=Q_{-}=0.3 \Gamma$ (tristable), $Q_{+}=Q_{-}=1.0 \Gamma$ (bistable). The equilibrium points satisfying $f_{\mu}\left(m_{z}\right)=0$ are stable (unstable) when $f_{\mu}^{\prime}\left(m_{z}\right)<0(>0)$. In the bistable case, for example, the point $m_{z}=0$ is unstable and $m_{z}= \pm 0.44$ are stable.

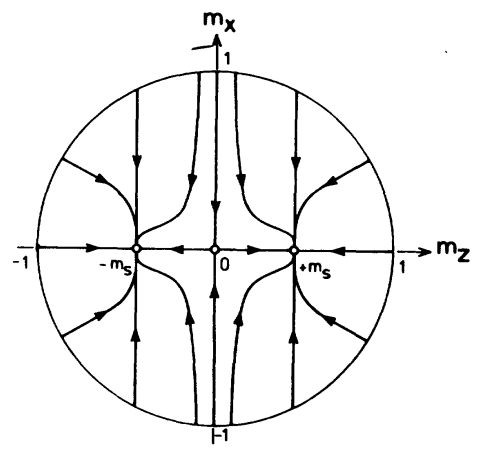

FIG. 4. Some trajectories of $\overrightarrow{\mathrm{m}}$ on the $\left(m_{z}, m_{x}\right)$ plane for the bistable case. The stable equilibrium point $\overrightarrow{\mathrm{m}}=\left(m_{s}, 0\right)$ corresponds to the $\sigma_{+}$state and $\overrightarrow{\mathrm{m}}=\left(-m_{s}, 0\right)$ corresponds to the $\sigma_{-}$state.

point $\vec{m}=0$ is unstable and the atomic spins orient parallel or antiparallel to the $z$ axis spontaneously. It is quite natural to expect that if $\Omega_{0} \neq 0$, the spontaneous magnetization continues to precess about the static magnetic field without decaying to $\overrightarrow{\mathbf{m}}=0$. To see the effect qualitatively we introduce the following equations:

$$
\begin{aligned}
& \frac{d m_{x}}{d t}=-\Omega_{0} m_{z}-\xi m_{x}, \\
& \frac{d m_{z}}{d t}=\Omega_{0} m_{x}-\eta m_{z}\left(m_{z}^{2}-m_{s}^{2}\right),
\end{aligned}
$$

where $\xi$ and $\eta$ are positive constants. The first terms of the right-hand side represent the precession about the $y$ axis and the second terms simulate the motion of $\vec{m}$ governed by Eqs. (4a) and (4c) in the absence of the static magnetic field. Namely, in the case of $\Omega_{0}=0$, Eqs. (10) have two stable equilibrium points $\left( \pm m_{s}, 0\right)$ and an unstable equilibrium point $(0,0)$ and give qualitatively the same flow as shown in Fig. 4. Equations (10) are quite similar to van der Pol's equations which describe the operation of electron tube oscillators. The difference is the presence of the terms $\xi m_{x}$ in Eq. (10a) but it can easily be eliminated by a variable transformation. By choosing suitable parameters, Eqs. (10) give a stable limit cycle on the $\left(m_{z}, m_{x}\right)$ plane. To confirm the oscillaton we solved Eqs. (4a) and (4c) numerically. Figure 5 gives an example of the trajectories, which starts from a point close to the origin, spirals out, and approaches to a limit cycle asymptotically. Two bends on the limit cycle are vestiges of the attractors which are located at the points $\left( \pm m_{s}, 0\right)$ when no magnetic field is applied. The velocity of the moving point becomes slower in the neighborhood of the bends, and therefore the period of rotation is longer than the 


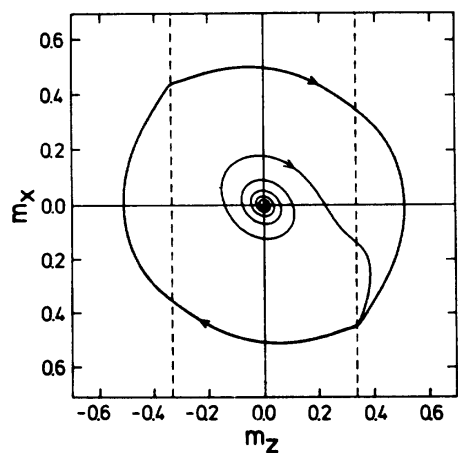

FIG. 5. Self-sustained precession of Bloch vector $\overrightarrow{\mathrm{m}}$. Parameters are $R=0.7, \Omega_{0}=50 \Gamma, 2 k_{0} L=-\pi+2 M \pi$, $2 \kappa L=1.5 \pi$, and $Q_{+}=Q_{-}=10 \Gamma$.

Larmor period $T_{0}=2 \pi / \Omega_{0}$. With an increase of $\Omega_{0}$, the period approaches to $T_{0}$ and the limit cycle becomes rounder. There is a critical value of $\Omega_{0}$ below which the trajectories are trapped to either of the attractors (for the parameters in Fig. 5, the critical value is about $40 \Gamma$ ). We note that the limit cycle lies in the real space, whereas in cases of other self-pulsing phenomena, it lies in a mathematically constructed space, namely, in a phase space.

We also calculated temporal behaviors of $\boldsymbol{P}_{ \pm}$ which are proportional to the intensities of the $\sigma_{+}$ and $\sigma_{-}$components of the transmitted light, respectively (Fig. 6). The oscillations in $\boldsymbol{P}_{ \pm}$are built up according to the growth of the trajectory in Fig. 5. In the steady state, alternative pulsations in $P_{ \pm}$are observed. In the half-cycle of $m_{z}>0$ $(<0), P_{+}\left(P_{-}\right)$is enhanced and $\left|m_{z}\right|$ is elongated through optical pumping. Thus the length of the Bloch vector $\overrightarrow{\mathrm{m}}$ is maintained against the thermal relaxations.

The two peaks in each pulse, one of which is sharp and the other broad, are explained as follows. For example $P_{+}$, which is a function of $m_{z}$,

${ }^{1}$ M. Kitano, T. Yabuzaki, and T. Ogawa, Phys. Rev. Lett. 46, 924 (1981).

${ }^{2}$ A. C. Tam and W. Happer, Phys. Rev. Lett. $\underline{\text { 38, }} 278$ (1977).

${ }^{3}$ A. Kastler, C. R. Acad. Sci. 252, 2396 (1961).

${ }^{4}$ S. L. McCall, Appl. Phys. Lett. 32, 284 (1978).

${ }^{5}$ A. Szöke, V. Daneu, J. Goldhar, and N. A. Kurnit,

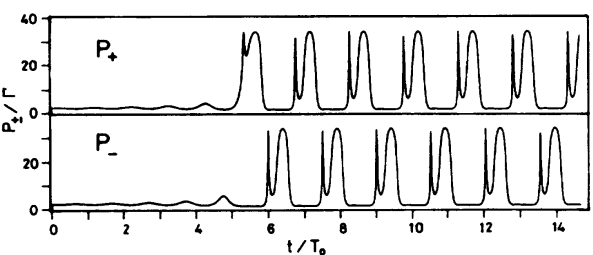

FIG. 6. Self-pulsing in $\sigma_{+}$and $\sigma_{-}$components of the transmitted light. Corresponding trajectory of $\vec{m}$ is shown in Fig. 5.

takes the maximum value on the dotted line in the half plane $m_{z}>0$ of Fig. 5. The trajectory crosses the line twice in a cycle, first rapidly in the upper half-plane and secondly slowly in the lower half-plane.

Finally, we propose an actual system which exhibits the phenomenon described above. The system is essentially the same as in Ref. 1, where sodium vapor was used as a nonlinear medium. The $D_{1}$ line $(589.6 \mathrm{~nm})$ is homogeneously broadened by filling a relatively high-pressure buffer gas (e.g., $\gamma_{a b} \sim 2 \mathrm{GHz}$ for 200 torr of helium), and the Doppler broadening can be neglected. The buffer gas also serves to prevent the hyperfine pumping in the ground state $3^{2} S_{1 / 2}$.

Thus, the behavior of the atom can be described substantially by the model depicted in Fig. 1. Taking $|\Delta| \sim 30 \gamma_{a b}$, vapor density $N \sim 3 \times 10^{12} \mathrm{~cm}^{-3}$, and $L \sim 10 \mathrm{~cm}$, we obtain the maximum phase shift $2 \kappa L \sim 5$. The spin relaxation rate $\Gamma$ is estimated to be $\sim 10^{3} \mathrm{~s}^{-1}$, and therefore the oscillation will be observed for the Larmor frequency $\Omega_{0}>40 \Gamma \sim 4 \times 10^{4} \mathrm{~s}^{-1}$, which corresponds to $H_{0} \gtrsim 10 \mathrm{mG}$. The required power density of a cw dye laser is of the order of $10 \mathrm{~mW} / \mathrm{mm}^{2}$.

One of us (M. K.) is a recipient of the Sakkokai Foundation Fellowship.
Appl. Phys. Lett. 15, 376 (1969).

${ }^{6}$ R. Bonifacio, L. A. Lugiato, and M. Gronchi, in Laser Spectroscopy $I V$, edited by $\mathrm{H}$. Walther and $\mathrm{K}$. W. Rothe (Springer, Berlin, 1979).

${ }^{7}$ H. G. Dehmelt, Phys. Rev. 105, 1924 (1957).

${ }^{8}$ W. Happer, Rev. Mod. Phys. 44, 169 (1972). 